\title{
Lead Site CV5RL
}

National Cancer Institute

\section{Source}

National Cancer Institute. Lead Site CV5RL. NCI Thesaurus. Code C90405.

A unipolar chest lead used mostly in large animals. Placed on the sixth ICS on the right side of the thorax along a line parallel to the level of the point of the elbow. 